Original paper

\title{
FRoG dose computation meets Monte Carlo accuracy for proton therapy dose calculation in lung
}

\author{
Giuseppe Magro $^{\text {a }}$, Stewart Mein ${ }^{\text {b,c, d,e }}$, Benedikt Kopp ${ }^{\text {b,c,d,e,f }}$, Edoardo Mastella ${ }^{a}$, \\ Andrea Pella $^{\mathrm{a}}$, Mario Ciocca ${ }^{\mathrm{a}}$, Andrea Mairani ${ }^{\mathrm{a}, \mathrm{b}, \mathrm{g}, *}$ \\ ${ }^{a}$ National Centre for Oncological Hadrontherapy (CNAO), Clinical Department, Pavia, Italy \\ ${ }^{\mathrm{b}}$ Clinical Cooperation Unit Translational Radiation Oncology, National Center for Tumor Diseases (NCT), Heidelberg University Hospital (UKHD) and German Cancer \\ Research Center (DKFZ), Heidelberg, Germany \\ ${ }^{c}$ Division of Molecular and Translational Radiation Oncology, Department of Radiation Oncology, Heidelberg Faculty of Medicine (MFHD) and Heidelberg University \\ Hospital (UKHD), Heidelberg Ion-Beam Therapy Center (HIT), Heidelberg, Germany \\ ${ }^{\mathrm{d}}$ German Cancer Consortium (DKTK) Core-Center Heidelberg, German Cancer Research Center (DKFZ), Heidelberg, Germany \\ ${ }^{\mathrm{e}}$ Clinical Cooperation Unit Radiation Oncology, Heidelberg Institute of Radiation Oncology (HIRO), National Center for Radiation Oncology (NCRO), Heidelberg \\ University and German Cancer Research Center (DKFZ), Heidelberg, Germany \\ ${ }^{\mathrm{f}}$ Faculty of Physics and Astronomy, Heidelberg University, Germany \\ ${ }^{\mathrm{g}}$ Heidelberg Ion-Beam Therapy Center (HIT), Department of Radiation Oncology, Heidelberg University Hospital, Heidelberg, Germany
}

\section{A R T I C L E I N F O}

\section{Keywords:}

Proton-therapy

Pencil-beam algorithm

GPU

Lung

\begin{abstract}
A B S T R A C T
Purpose: To benchmark and evaluate the clinical viability of novel analytical GPU-accelerated and CPU-based Monte Carlo (MC) dose-engines for spot-scanning intensity-modulated-proton-therapy (IMPT) towards the improvement of lung cancer treatment.

Methods: Nine patient cases were collected from the CNAO clinical experience and The Cancer Imaging Archive4D-Lung-Database for in-silico study. All plans were optimized with 2 orthogonal beams in RayStation (RS) v.8. Forward calculations were performed with FRoG, an independent dose calculation system using a fast robust approach to the pencil beam algorithm (PBA), RS-MC (CPU for v.8) and general-purpose MC (gp-MC). Dosimetric benchmarks were acquired via irradiation of a lung-like phantom and ionization chambers for both a single-fielduniform-dose (SFUD) and IMPT plans. Dose-volume-histograms, dose-difference and $\gamma$-analyses were conducted. Results: With respect to reference gp-MC, the average dose to the GTV was $1.8 \%$ and 2.3\% larger for FRoG and the RS-MC treatment planning system (TPS). FRoG and RS-MC showed a local $\gamma$-passing rate of $\sim 96 \%$ and $\sim 93 \%$. Phantom measurements confirmed FRoG's high accuracy with a deviation $<0.1 \%$.

Conclusions: Dose calculation performance using the GPU-accelerated analytical PBA, MC-TPS and gP-MC code were well within clinical tolerances. FRoG predictions were in good agreement with both the full gp-MC and experimental data for proton beams optimized for thoracic dose calculations. GPU-accelerated dose-engines like FRoG may alleviate current issues related to deficiencies in current commercial analytical proton beam models. The novel approach to the PBA implemented in FRoG is suitable for either clinical TPS or as an auxiliary doseengine to support clinical activity for lung patients.
\end{abstract}

\section{Introduction}

With lung cancer as the leading cause of cancer-related deaths worldwide, innovative and more effective therapies, such as proton therapy (PT), are urgently needed. Improvement in tumor targeting with increased healthy tissue sparing compared to conventional photon beams is especially critical in the treatment of inoperable lung tumors
[1]. Accordingly, interest in PT application is on the rise for lung cancer, one of several disease sites where proton beams show promise in improving clinical efficacy $[2,3]$, considering the potential for healthy lung sparing and/or the reduction of the dose to the heart [4].

In practice, several dosimetric limitations and sources of uncertainty arise from treatment planning (TP) to delivery with proton beams for lung cancer. The favorable physical characteristics themselves, e.g.

\footnotetext{
* Corresponding author at: National Centre for Oncological Hadrontherapy (CNAO), Clinical Department, Pavia, Italy.

E-mail address: andrea.mairani@med.uni-heidelberg.de (A. Mairani).
} 
Bragg-peak with reduced lateral penumbra, make PT an ideal candidate for high-precision treatment delivery in heterogeneous anatomical sites such as the lung, but may also make these treatments increasingly prone to uncertainties in range and temporal effects due to breathing/organ motion $[5,6]$. Clinical consideration through increased target margins, 4D-robust TP, gating and/or rescanning delivery techniques, has demonstrated promising results to reduce sensitivity to delivery uncertainties and/or interplay effects [7,8]. Nonetheless, significant discrepancy between planned and delivered dose may occur if dose algorithms are not properly managed by the clinical TP systems (TPSs). For instance recent works highlight relevant dosimetric differences between measured and predicted dose by conventional approaches in most commercial TPSs [9], in part due to improper modeling of radiation transport in highly heterogeneous tissues such as the lung, consisting of complex bone-air-tissue interfaces, anatomic/geometric complexities and sub-voxel Hounsfield-Units (HU) variations. "Monte Carlo (MC)versus-analytical algorithm" remains a common debate topic and correspondingly, thoracic subcommittees aim to develop urgently needed consensus and guidelines for quality assurance (QA), TP and delivery of particle therapy for thoracic malignancies [10].

Excluding uncertainties attributed to the breathing cycle/dose delivery system, this work specifically focuses on inherent accuracy of an analytical dose algorithm in thoracic regions, which in most other clinical scenarios can provide acceptable plan calculations within wellestablished tolerances. Despite being subject to scrutiny, the pencil beam algorithm (PBA) in PT provides fast speeds at the potential sacrifice of accuracy in complex tissue inhomogeneities. The gold standard for accuracy is the MC simulation and related codes are only recently introduced to the clinics, fostered by past and on-going efforts to develop clinical dose engines [11,12].

In the case of thoracic treatment sites, several studies demonstrate limitations of clinical TPSs where accuracy is critical [13,14]. Most notably, investigations with anthropomorphic lung phantoms suggest that application of commercial TPSs using analytical algorithms for the treatment of lung tumors should be deemed unfit for clinical use or used with extreme caution [15]. The lateral dose distribution computed by PBA in the lung and in bone interfaces may reach a level of inaccuracy around $30 \%[16,17]$, while commercial MC-based TPSs can improve the accuracy of dose calculation in the lung/bone interfaces or through inhomogeneities within $\sim 5 \%[12,17]$. Despite being considered clinically tolerable, another source of uncertainty in proton therapy of lung originates from the heterogeneous structure of the lung itself, which leads to a degradation of the Bragg peak and a wider distal fall-off [18].

Several other works have investigated the accuracy of analytical algorithms and/or MC codes in clinically-relevant scenarios, especially in head-and-neck phantoms [16,17,19-21], however, few studies perform comprehensive testing for thorax-based treatments beyond commercial approaches.

In this work, dose calculation for lung cancer patients is investigated using the FRoG system, a GPU-accelerated dose calculation platform for particle therapy with both an enhanced physics-engine and rapid computation speed made possible via task-parallelization for multiple particle species [22,23]. The capabilities and limits of the FRoG approach are tested via dose calculation for thoracic malignancies. Through extensive benchmarking against in-silico references (clinical TPS and general purpose (gp)-MC simulation), as well as experimental validation through end-to-end QA tests in an in-house built heterogeneous phantom equipped with ionization chambers (ICs), we verify whether analytical methods for PT dose calculation are indeed unsuitable for clinical activity.

Furthermore, we explore dose-averaged linear energy transfer $\left(\mathrm{LET}_{D}\right)$ in the context of lung cancer patients, to assess flexibility and feasibility of relating innovative bio-effect quantities with clinical efficacy. This analysis has yet to be presented in the literature. Further efforts are made here to validate FRoG as a secondary dose engine for supporting clinical decision-making at CNAO, the Heidelberg Ion-beam
Therapy-center (HIT) and the Aarhus Danish Center for Proton Therapy, where the platform has been installed for rapid physical, $\mathrm{LET}_{D}$, and biodose prediction. Here, state-of-the-art GPU-accelerated analytical and CPU-based MC dose engines are rigorously tested in scenarios where conventional approaches to clinical dose calculation often fail.

\section{Material and methods}

\subsection{Patients selection}

A total of 9 non-small-cell lung cancer (NSCLC) patients, with tumors differing in stage (I to III) and gross tumor volume (GTV) size (range = 6-160 cc, median = $19 \mathrm{cc}$ ), were randomly selected from The Cancer Imaging Archive-4D Lung Database [24] and from the CNAO clinical experience. For the selected cohort, the average minimum and maximum target-to-lung wall distance, expressed as point-to-point surface separation, was $0 \mathrm{~cm}$ and $20.5 \mathrm{~cm}$, respectively. On average, GTVs were surrounded by $6.5 \mathrm{~cm}$ of lung tissue, with one GTV only $(6.12 \mathrm{cc})$ being completely detached from the lung wall $(2 \mathrm{~cm}$ and $17 \mathrm{~cm}$ were its minimum and maximum distance). All patient calculations performed in this study (FLUKA gp-MC, FRoG and RayStation ${ }^{\circledR}$ v.8-MC (RS-MC) TPS) considered dose-to-water $\left(D_{w}\right)$ as opposed to dose-to-medium following clinical procedure.

\subsection{TPS settings}

Intensity modulated proton therapy (IMPT) was employed during plan optimization of the lung patient data, using 2 orthogonal ports and scanning parameters as established by CNAO clinical protocols [25]. The optimization strategy using 4D-computed tomography (CT)-scans is detailed in a previous work [26]. Prescription dose was set to $60 \mathrm{~Gy}$ (Relative Biological Effectiveness, RBE $=1.1$ ), delivered in 10 equal fractions to the GTV in terms of median dose $\left(D_{50}\right)$. The RS v.8-MC clinical v.4.2 was used as dose engine for the optimization problem, as recommended by recent studies $[15,27]$. A robust optimization strategy, accounting for a set of scenarios which reflect delivery and target motion uncertainties were incorporated in the problem formulation [26] and a minimax optimization was performed [28]. In this study, all forward calculations were conducted with FRoG and the gp-MC using the end-ofexhale phase as the reference (static) patient anatomy, neglecting the beam distortion effects from the breathing cycle to solely investigate dose engine performance. Ten-thousand ions-per-spot were selected at the optimization stage and an estimated average statistical error per voxel $<0.5 \%$ for the final dose distribution was required to the system. The error is estimated over all voxels having a dose above $50 \%$ of the maximum dose per beam (RayStation ${ }^{\circledR}$ Reference Manual). The dose grid resolution was fixed to $2 \times 2 \times 2 \mathrm{~mm}^{3}$, which coincided with the particles transport grid and the CNAO-specific CT calibration curve, used routinely for organ motion-based acquisitions, was assigned to the CT matrix.

\subsection{FRoG settings}

In previous works, FRoG demonstrated excellent performance in terms of both accuracy and speed when compared to gp-MC, clinical TPS predictions and measurements too. Here, those verified settings, i.e. PB splitting multiplicity of $\sim 350$ and triple-Gaussian lateral dose parameterization, were implemented for investigating dose calculation in heterogeneous conditions of the lung. FRoG is composed of an analytical PBA algorithm, sampled from a dosimetric database derived from MC simulation and performs rapid computations via GPU-accelerated engines (e.g. raytracing, dose calculation kernels, etc.).

\section{4. $g p-M C$ settings}

The FLUKA gp-MC simulation code [29] was selected as the gold 
standard reference for accuracy during comparison of patient dose calculation of GPU-based engines adopted by FRoG and by the RS-MC TPS. FLUKA has been supporting clinical and research activities at CNAO since 2011 and it has been extensively validated against clinical data [25], therefore providing a valuable reference for the benchmarking of such challenging scenarios. For a straightforward comparison between the performances of the above-mentioned systems and the gp-MC dose calculations in the lung tissue, HU-dependent tuning of the stopping power computation was introduced during runtime, which was specific for the lung, to force the gp-MC to reproduce the same CT calibration curve as used by FRoG and the RS-MC TPS [30]. The default hadrontherapy setting was selected as the main physics package for lung calculations and the same TPS dose grid resolution was used for plan dose recalculations. Five thousand primary protons per PB on a $2 \times 2 \times$ $2 \mathrm{~mm}^{3}$ voxel dose grid were selected to reach a mean statistical uncertainty of $\sim 0.5 \%$ for each voxel in the GTV [31].

\subsection{Phantom study}

For dosimetric validation, a cork box-phantom $\left(15 \times 15 \times 15 \mathrm{~cm}^{3}\right)$ $[26,32]$ was assembled in-house to mimic human lung tissue, with a $4 \times$ $4 \times 4 \mathrm{~cm}^{3}$ water-filled cavity as the target (Fig. 1). Five calibrated PinPoint ICs (PTW@ model 31015) connected to a multi-channel precision electrometer (PTW $\odot$ Multidos) were placed inside the water cavity to measure absorbed $D_{w}$, in accordance with the IAEA TRS 398 code of practice. One IC was placed at isocenter (geometric center of water cavity), while the remaining ICs were displaced within the target and spaced apart (honeycomb scheme) to avoid IC-IC perturbations. For end-to-end testing, a CT scan was acquired following clinical protocol and two plans were optimized on the RS-MC TPS (prescription dose of 6 Gy(RBE) in a single fraction): first, a 2-orthogonal beams IMPT plan (infield inhomogeneity testing) and second, a single field uniform dose (SFUD) plan, both with a $3 \mathrm{~cm}$-thick range shifter placed at $10 \mathrm{~cm}$ distance from the phantom surface. Each IC was contoured on the CT scan and a cross-check was performed on the TPS calculated volume to match the sensitive volume of the detector. Plans were delivered in a fixedbeam room at CNAO, using the horizontal beam port only.

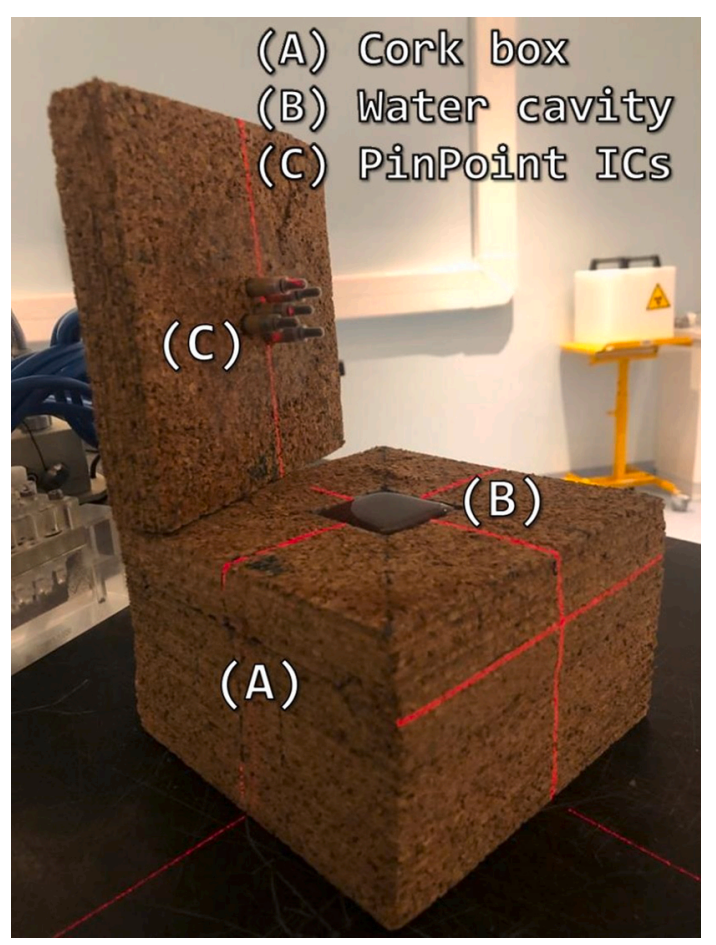

Fig. 1. Lung phantom experimental setup used for the end-to-end test of FRoG.

\subsection{Dose-averaged LET maps}

Further investigations with the FRoG engine involved selection and testing of the 2 patient cases with the smallest and largest target (GTV: $\sim 6 \mathrm{cc}$ and $\sim 160 \mathrm{cc}$ ) to evaluate resultant $\mathrm{LET}_{D}$ distributions from RS-MC optimized plans. $\mathrm{LET}_{D}$ maps were calculated with PB-type (MC-derived) $\mathrm{LET}_{D}$-kernels, as widely described in [22].

\subsection{Data analysis}

Dose ratios for FRoG and RS-MC TPS to gp-MC were recorded for the following plan metrics: GTV $D_{50}$, near-to-maximum dose $\left(D_{2}\right)$, near-tominimum dose $\left(D_{98}\right)$ and the average dose to the ipsilateral lung ( $D_{\text {lung }}$ ). The dose indices $D_{2}$ and $D_{98}$ on the target volume were selected as recommended by the ICRU Report 93 for quantification and assessment of coverage, while the median dose was used to evaluate to what degree the prescribed dose level was reached by the calculation engine. $D_{\text {lung }}$ was chosen to give an estimate of the average dose level in the surrounding of the target. To quantify homogeneity of the dose distribution within the GTV at dose-volume histogram (DVH) level, the Homogeneity Index $(\mathrm{HI})$ difference $(\Delta \mathrm{HI})$ (where HI[\%] $\left.=\left(D_{2}-D_{98}\right) / D_{50}\right)$ was computed. The choice of the MC histories (both for the gp-MC and for the RS-MC) allowed us to safely use the above mentioned thresholds to evaluate the steepness of the DVH, which, in general, is highly dependent on the number of simulated primary particles. For avoidance of software-related biases on the computation of the DVHs, all dose matrices were loaded on the TPS to extract the dose statistics used for the data analysis.

In addition, using the PTW $\odot$ plan verification software Verisoft ${ }^{\circledR}$ v.6.0, differences in the dose distributions were assessed via 3D$\gamma$-analysis [33], reported as local $\gamma$ passing rate (L $\gamma$-PR), defined as the percentage of points with $\gamma \leq 1$. For both FRoG and RS-MC, evaluation was conducted under a $1 \mathrm{~mm} / 3 \%$ distance-to-agreement/local dosedifference (DTA/LDD) criterion with a 5\% low dose-threshold (DT), with gp-MC as the reference. All datasets were normalized to the prescription dose-per-fraction, i.e. $6 \mathrm{~Gy}(\mathrm{RBE})=100 \%$. The DTA value of 1 $\mathrm{mm}$ reflects the CT axial resolution $(0.88 \mathrm{~mm})$, while $\mathrm{DT}=5 \%$ allowed suppression of doses below clinical relevance for these specific cases. Finally, the LDD $=3 \%$ met the recommendation of the AAPM TG 218 [34]. The strict $\gamma$-criteria selected were necessary to appropriately assess dosimetric requirements for thoracic-based treatment sites. The tolerance level for the $\mathrm{L} \gamma$-PR was fixed to $90 \%$ (action level), corresponding to non-optimal but acceptable plans. A global $\gamma$ passing rate (G $\gamma$-PR) (with global dose-difference, GDD $=3 \%$ of $6 \mathrm{~Gy}(\mathrm{RBE})$ ) was considered to evaluate how possible local dose discrepancies, detectable via a poor $\mathrm{L} \gamma$-PR, might prevent FRoG and RS-MC dose distributions from meeting the tolerance level of $95 \%$.

For the phantom study, measured IC dose was compared against the average dose $\left(D^{-}\right)$as computed by FRoG and the TPS for each region of interest (IC active volume) via an average dose ratio over the 5 ICs. Additionally, to explore the worst case scenario for potentially nonuniform beams, more in-depth evaluation was conducted for the IMPT plan by determining the global absolute percent dose deviation $|\% \Delta|$ between each system's prediction and the corresponding measured dose.

Lastly, $\mathrm{LET}_{D^{-}}$volume histograms ( $\mathrm{LVHs}$ ) were computed for FRoG and for the gp-MC LET $\mathrm{L}_{D}$ matrices, to provide further benchmarks of the analytical $\mathrm{LET}_{D}$ distributions for the thoracic sites. Average and near-tomaximum $\mathrm{LET}_{D}$ from LVH (LET $\mathrm{Lvg}_{\text {g }}$ and $\mathrm{LET}_{2}$ ) were computed for the ipsilateral lung, GTV and a ring expansion of the GTV (margins increased by $0.5 \mathrm{~cm}$ to $2.5 \mathrm{~cm}$ ), with the latter to evaluate $\operatorname{LET}_{D}$ within the vicinity of the target. 


\section{Results}

\subsection{Recalculations of patient plans}

\subsubsection{DVH analysis}

Table 1 summarizes the DVH statistics as listed in Data analysis for the GPU-based dose engine FRoG and the CPU-based RS-MC, reported in terms of dose ratios against the gp-MC code. In Fig. 2, two representative patient cases are displayed with a FRoG dose map, alongside DVH and dose profiles comparing gp-MC, FRoG and the RS-MC TPS.

In general, DVH metrics indicate that both dose engines slightly overestimate the gp-MC prediction by $\sim 2 \%$ (see $D_{50}$ ), with the largest discrepancies observed for the near-to-minimum and near-to-maximum doses. Notably, the results in Table 1 demonstrate that $D_{2}$ and $D_{98}$ differ from the reference by approximately the same \%-value and, correspondingly, the almost zero value observed for $\Delta \mathrm{HI}_{\mathrm{FRoG}-\mathrm{gp}-\mathrm{MC}}$ is consistent. $\Delta \mathrm{HI}_{\mathrm{RS}-\mathrm{MC}-\mathrm{gp}-\mathrm{MC}}$ has also effectively negligible deviations. Moreover, absorbed dose to the surrounding lung tissue is well reproduced by both FRoG and the RS-MC TPS compared with the gp-MC, further demonstrated by DVH and line profiles.

\subsubsection{Gamma analysis}

Results of 3D- $\gamma$-analyses are presented in Fig. 3, in terms of $\mathrm{G} \gamma$-PR and $\mathrm{L} \gamma$-PR, respectively. The bar-chart presents the patient cohort by increasing GTV, although there is no clear evidence of volume dependency on the passing rate: visualization of both FRoG and RS-MC TPS depicts a nearly random fluctuation of bar height regardless of the target size. Final $\gamma$-PRs averaged over the entire cohort follow with the standard deviation and range of variation:

G $\gamma$-PR

o FRoG vs. gp-MC: $(97.0 \pm 1.4) \% \in[95.1 ; 99.1] \%$;

o RS-MC TPS vs. gp-MC: $(97.8 \pm 1.2) \% \in[95.0 ; 98.8] \%$;

- $\mathrm{L} \gamma \mathrm{-PR}$

o FRoG vs. gp-MC: $(95.8 \pm 1.8) \% \in[93.3 ; 98.2] \%$;

o RS-MC TPS vs. gp-MC: $(92.7 \pm 2.0) \% \in[88.6 ; 95.5] \%$;

\subsection{Experimental validation}

SFUD and IMPT plans were delivered within an end-to-end workflow for the experimental validation of FRoG, RS-MC TPS and gp-MC in thoracic-like settings. Table 2 reports the dose engine-to-data ratios averaged over the 5 ICs placed in the in-house built cork box-phantom.

\section{3. $L E T_{D}$ distributions}

As reported in Table 3, irrespective of target volume and thickness of traversed lung tissue, FRoG and FLUKA $\mathrm{LET}_{D}$ predictions are in agreement within $0.1 \mathrm{keV} / \mu \mathrm{m}$ for the GTV $\left(\sim 3 \%\right.$ of GTV $\left.\mathrm{LET}_{\mathrm{avg}}\right)$ and within

Table 1

FRoG-to-gp MC and RS-MC TPS-to-gp MC ratios for $D_{50}, D_{98}$, and $D_{2}$. HIdifferences and $D_{\text {lung }}$ have been also reported.

\begin{tabular}{|c|c|c|c|}
\hline & & $\begin{array}{l}\text { FRoG/gp-MC } \\
\text { Average } \pm 1 \sigma \\
\text { (min.;max.) }\end{array}$ & $\begin{array}{l}\text { RS-MC TPS/gp-MC } \\
\text { Average } \pm 1 \sigma \\
\text { (min.;max.) }\end{array}$ \\
\hline \multirow[t]{4}{*}{ GTV } & $D_{50}$ & $\begin{array}{l}1.016 \pm 0.009 \\
(1.003 ; 1.029)\end{array}$ & $\begin{array}{l}1.022 \pm 0.006 \\
(1.013 ; 1.032)\end{array}$ \\
\hline & $D_{2}$ & $\begin{array}{l}1.024 \pm 0.010 \\
(1.011 ; 1.042)\end{array}$ & $\begin{array}{l}1.030 \pm 0.008 \\
(1.019 ; 1.043)\end{array}$ \\
\hline & $D_{98}$ & $\begin{array}{l}1.026 \pm 0.010 \\
(1.016 ; 1.040)\end{array}$ & $\begin{array}{l}1.026 \pm 0.012 \\
(1.009 ; 1.044)\end{array}$ \\
\hline & $\Delta \mathrm{HI}$ & $\begin{array}{l}(0.1 \pm 0.9) \% \\
(-0.8 ; 1.7) \% \\
(\text { FRoG - gp-MC) }\end{array}$ & $\begin{array}{l}0.7 \pm 0.8 \% \\
(-0.6 ; 2.2) \% \\
(R S-M C-g p-M C)\end{array}$ \\
\hline Ipsilateral lung & $D_{\text {lung }}$ & $\begin{array}{l}0.994 \pm 0.019 \\
(0.956 ; 1.016)\end{array}$ & $\begin{array}{l}0.998 \pm 0.017 \\
(0.970 ; 1.017)\end{array}$ \\
\hline
\end{tabular}

$0.5 \mathrm{keV} / \mu \mathrm{m}\left(\sim 7 \%\right.$ of lung $\left.\mathrm{LET}_{2}\right)$ for the other analyzed non-target structures.

In Fig. 4, $\mathrm{LET}_{D}$ maps and LVHs plots (GTV, wall and ipsilateral lung) for two representative lung patient cases are presented for the gp-MC and FRoG system.

\subsection{Calculation time}

RS-MC, the gp-MC and FRoG are installed on separate platforms and run on different hardware. Hence a fair comparison among the three systems is not justified in terms of calculation speed. Moreover, each code manages radiation transport and dose scoring parameters differently, as addressed in the Material and Methods section: the gp-MC and FRoG perform the particle transport on the native CT voxels and calculate the dose on the $2 \mathrm{~mm}$ resolution dose grid; the RS-MC on the other hand works on a transport grid identical to the dose calculation grid (RayStation ${ }^{\circledR}$ Reference Manual). Despite these technical differences, a brief analysis on the computation time was performed.

For the studied patient cohort, the average computation time per primary was about $8 \mathrm{~ms}$ for the gp-MC, resulting in an overall computation time on a cluster with 24 CPUs (Intel Xeon CPU E5-2660 v3 at $2.60 \mathrm{GHz}$ ) of about 1.5 to $12 \mathrm{~h}$ per beam, for the 6 and $160 \mathrm{cc}$ GTV, respectively.

For maximum performance with TPS v.8, computations were performed using hardware specifically recommended by RaySearch Laboratories $\odot$ and available at our clinic for its CPU-based proton MC dose engine, i.e. Intel Xeon CPU E5-2630 v4 at $2.20 \mathrm{GHz}$ (2 processors, 10 cores each). Computation times for the final dose calculation were $20 \mathrm{~s}$ per beam, on average. Computation time was recorded when calling the ComputeDose built-in function, i.e. the so-called "dose finalization", which includes the output of both a physical and a fixed-RBE( $=1.1)$ dose matrix.

In the context of FRoG runtimes, Choi et al. [22] previously demonstrated a time reduction of about 3.5-fold when moving, for example, from a consumer grade GPU card NVIDIA GeForce GTX 1080 Ti to a high-end graphics card NVIDIA Tesla V100, which is the best available configuration where the FRoG code is running so far. Calculation times ranged from 3.5 to $11.5 \mathrm{~s}$ per beam, when using NVIDIA Tesla V100 GPU and monitoring the time required for the handling of the dose kernel only, to be fairly comparable against the TPS. Within the overall calculation time, FRoG produces the output for physical dose, biological dose assuming a fixed-RBE, biological dose using a variableRBE model and a $\operatorname{LET}_{D}$ matrix (i.e. two more than the TPS output).

\section{Discussion}

Through systematic evaluations of PT dose calculation in thoracic treatment sites, we provide benchmarks for an innovative approach of the PBA as well as both clinical and research-based MC systems. In the context of results available in recent literature on dosimetric investigations using lung phantoms, this study demonstrated substantially higher PRs and lower dose deviations for an analytical system in spite of the relatively strict $\gamma$-criteria. We believe several points regarding lung dose calculation need formal clarification and here we will shed light on potential causes for deviations commonly observed with commercial systems, as well as suggested next steps for collaboration with industry towards improved TP for thoracic cancers.

First, a brief technical aside: higher deviations in $D_{98}$ and $D_{2}$ were observed for both FRoG and RS-MC in the DVH analysis against reference gp-MC. Apart from possible explanations like statistical fluctuation exhibited by MC engines [35,36], the discrepancy on the coverage of the $98 \%\left(D_{98}\right)$ of the GTV affected the smallest geometry available for the selected database. This patient case, in particular, exhibited also higher GTV $D_{50}$ ratios (FRoG $=1.029$, RS-MC $=1.032$ ), with overestimations by both dose engines $\sim 3 \%$ (see Fig. 2, bottom panels): this may confirm that deviations can be attributed to challenges arising from small target 

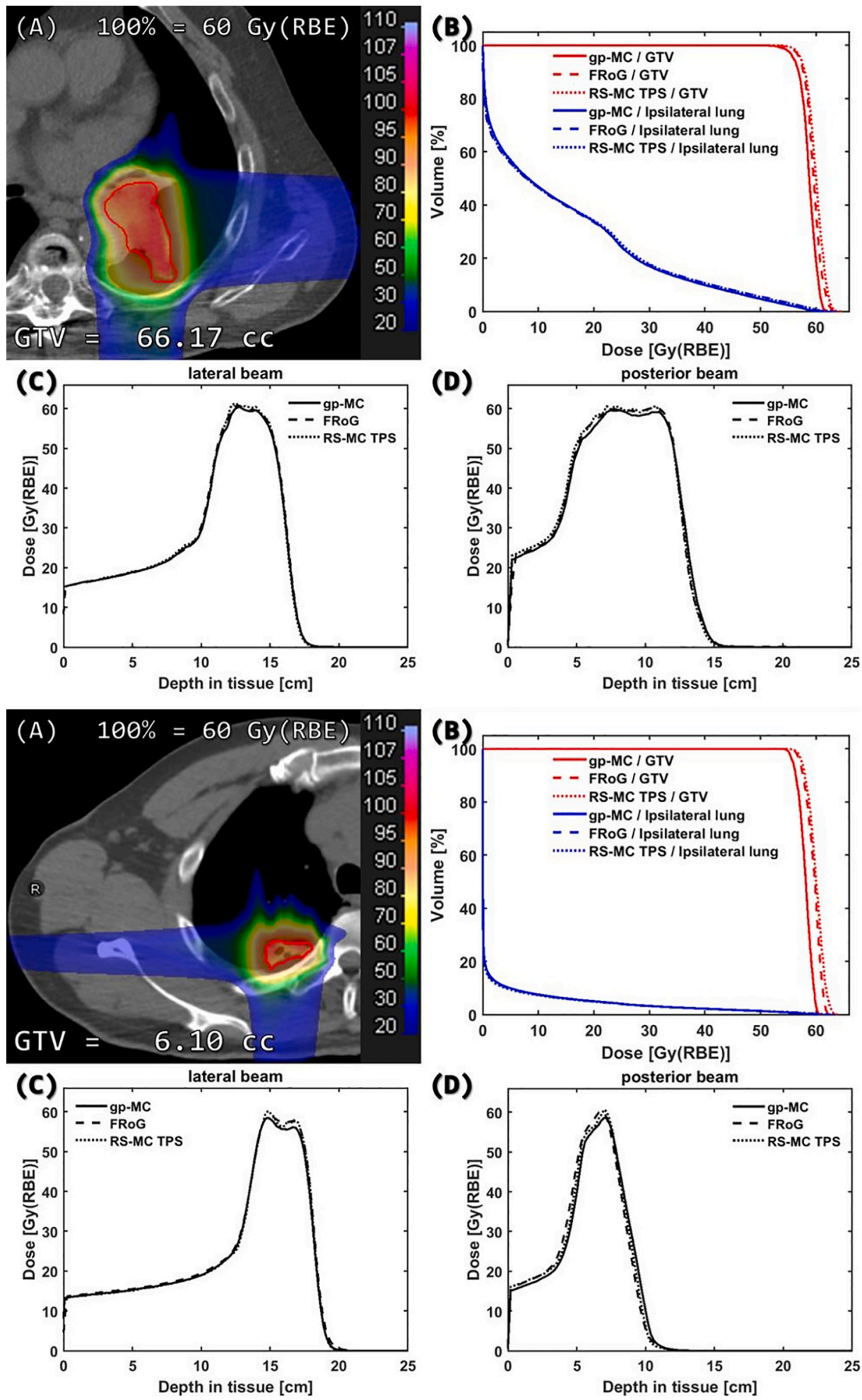

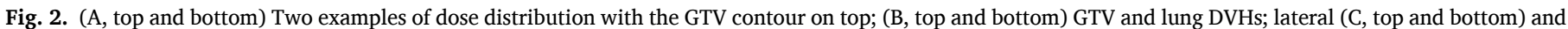
posterior ( $\mathrm{D}$, top and bottom) beam longitudinal profiles along their central axis.

volumes ( $\sim 6 \mathrm{cc})$, which may amplify possible initial discrepancies on the parameterization of the beam lateral spread in air throughout the beamline. In fact, thoracic treatment sites often host small targets with densities higher than the surroundings normal tissues and the lowdensity thoracic tissues may prevent the beam from spreading enough to smear out potential differences inherited from the patient entrance surface.
Overall, FRoG matches well with the gp-MC predictions. Considering all resultant values extracted from the DVHs (Table 1), FRoG reproduces gp-MC within $2.2 \%$, on average. Limiting analysis on the GTV, $\Delta \mathrm{HI}$ was consistent between both the dose engines, with respect to gp-MC baseline: $D^{-}$to the GTV was $1.8 \%$ and $2.3 \%$ greater for FRoG and the RS-MC TPS, respectively.

For both the dose engines, the average dose to the $D_{\text {lung }}$ agreed 
(A)

$\square$ FRoG $\square$ RS-MC TPS
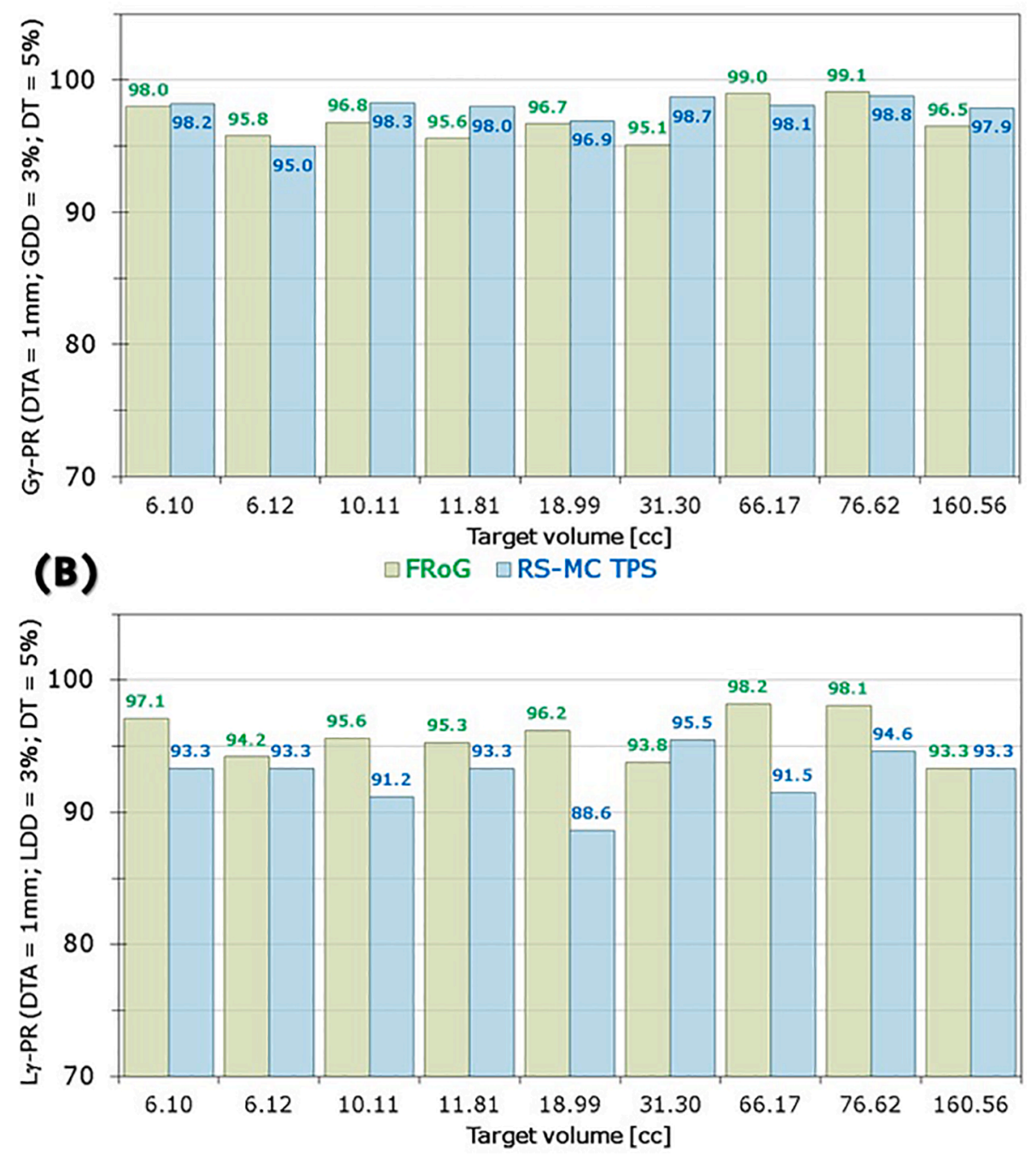

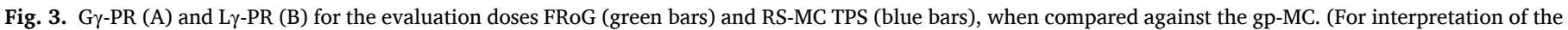
references to colour in this figure legend, the reader is referred to the web version of this article.)

Table 2

Dose ratios of FRoG, RS-MC TPS and gp-MC against measurements, reported as average value over 5 calibrated ICs for SFUD and IMPT plans.

\begin{tabular}{llll}
\hline & $\begin{array}{l}\text { gp-MC/Data } \\
\text { Average } \pm 1 \sigma \\
\text { (min.;max.) }\end{array}$ & $\begin{array}{l}\text { FRoG/Data } \\
\text { Average } \pm 1 \sigma \\
\text { (min.;max.) }\end{array}$ & $\begin{array}{l}\text { RS-MC TPS/Data } \\
\text { Average } \pm 1 \sigma \\
\text { (min.;max.) }\end{array}$ \\
\hline SFUD & $\begin{array}{l}1.002 \pm 0.017 \\
(0.974 ; 1.019)\end{array}$ & $\begin{array}{l}1.007 \pm 0.018 \\
(0.975 ; 1.021)\end{array}$ & $\begin{array}{l}1.001 \pm 0.016 \\
(0.977 ; 1.019)\end{array}$ \\
IMPT & $0.988 \pm 0.041$ & $1.000 \pm 0.046$ & $0.987 \pm 0.043$ \\
& $(0.940 ; 1.051)$ & $(0.977 ; 1.073)$ & $(0.941 ; 1.053)$ \\
\hline
\end{tabular}

Table 3

$\mathrm{LET}_{\text {avg }}$ and $\mathrm{LET}_{2} \mathrm{LVH}$ parameters extracted from FRoG and FLUKA LET ${ }_{D}$ maps. Data in $\mathrm{keV} / \mu \mathrm{m}$.

\begin{tabular}{|c|c|c|c|c|c|c|c|}
\hline \multirow{2}{*}{$\begin{array}{l}\text { GTV } \\
\text { volume }\end{array}$} & \multirow[t]{2}{*}{ LET } & \multicolumn{2}{|l|}{ GTV } & \multicolumn{2}{|c|}{ Wall 0.5-2.5 } & \multicolumn{2}{|c|}{ Ipsilateral lung } \\
\hline & & FRoG & gp-MC & FRoG & gp-MC & FRoG & gp-MC \\
\hline \multirow[t]{2}{*}{$6.10 \mathrm{cc}$} & $\mathrm{LET}_{\mathrm{avg}}$ & 3.4 & 3.3 & 3.5 & 3.3 & 0.5 & 0.5 \\
\hline & $\mathrm{LET}_{2}$ & 4.1 & 4.2 & 8.4 & 8.4 & 6.7 & 6.8 \\
\hline \multirow[t]{2}{*}{$160.56 \mathrm{cc}$} & $\mathrm{LET}_{\text {avg }}$ & 2.8 & 2.7 & 3.0 & 2.8 & 1.1 & 1.0 \\
\hline & $\mathrm{LET}_{2}$ & 3.7 & 3.7 & 7.0 & 6.9 & 6.9 & 6.4 \\
\hline
\end{tabular}

within $0.4 \%$, with a relative standard deviation of the $D_{\text {lung-ratio }}<2 \%$ for both the dose engines.

Considering the challenging nature of thoracic treatments, a $\mathrm{G} \gamma$ analysis with distance-to-agreement DTA $=1 \mathrm{~mm}$, global dosedifference GDD $=3 \%$ and dose-threshold DT $=5 \%$ may be considered sufficiently strict for validation purposes, considering the $5 \mathrm{~mm} / 7 \%$ criteria applied in recent works [15]. In this regard, Fig. 3(A) visibly demonstrates that all patient plans in the investigated cohort recalculated by FRoG satisfy the clinically optimal 95\%-tolerance level. No failures were detected for the RS-MC TPS as well, with the totality of cases above the 95\%-tolerance level. In turn, FRoG and RS-MC TPS exhibited comparable dose calculation performance.

Investigations were additionally conducted by adopting local dose normalization for the $\gamma$-analysis (Fig. 3(B)), a stringent method to validate dosimetric performances of FRoG specifically in lower dose areas. While maintaining the same evaluation criteria, RS-MC TPS exhibited slightly poorer performances when compared to local outcomes for the reference gp-MC. The observed $\mathrm{L} \gamma$-PR, in fact, lowers to a mean value of $(92.7 \pm 2.0) \%$, with only a single patient case with results above the 95\%-tolerance level. FRoG performs better against the gp-MC, even locally, with a mean $\mathrm{L} \gamma$-PR of $(95.8 \pm 1.8) \%$. Interestingly, for FRoG, $100 \%$ of patients exceed the $\mathrm{L} \gamma$-PR $>90 \%$, with 2 patients (among the 

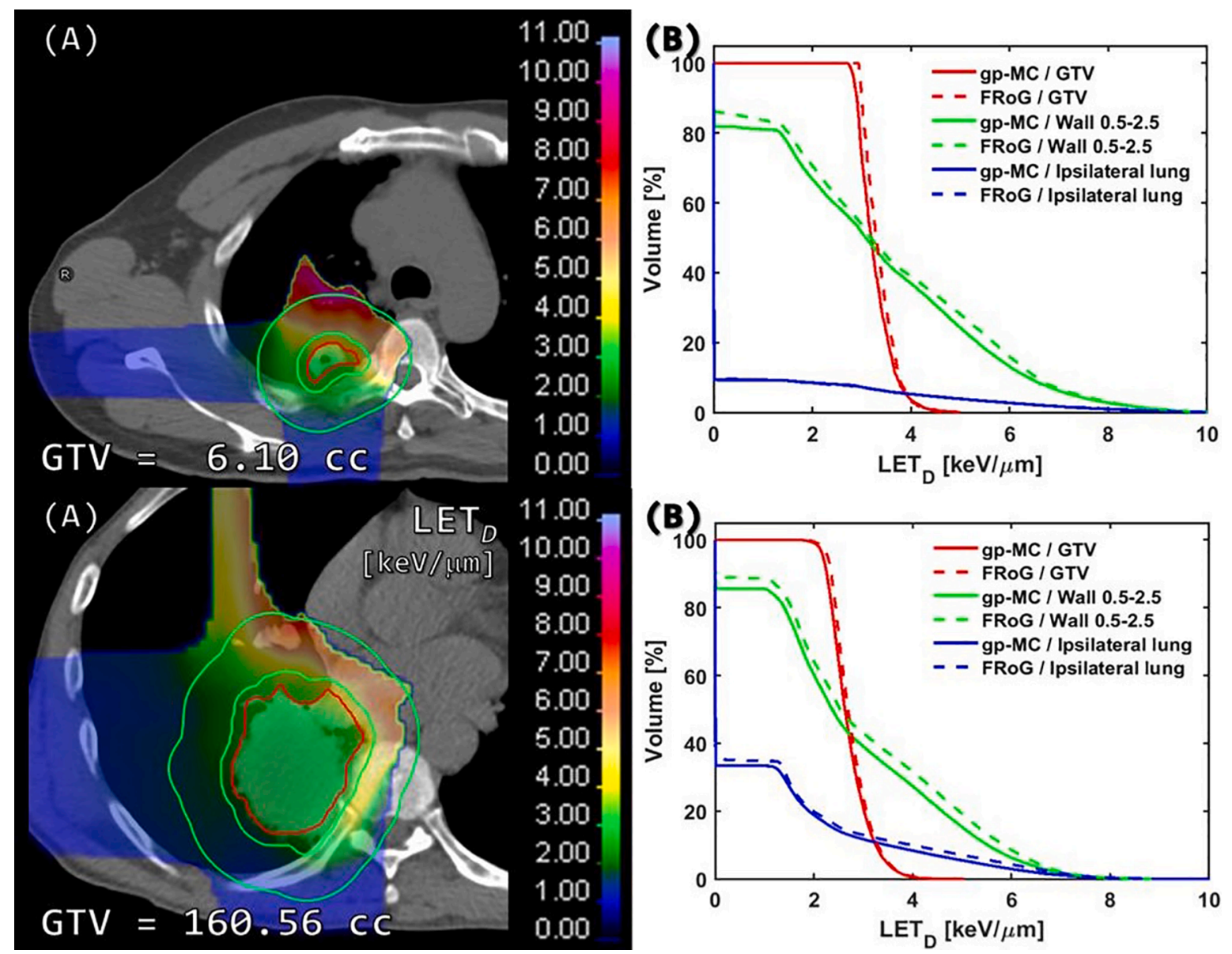

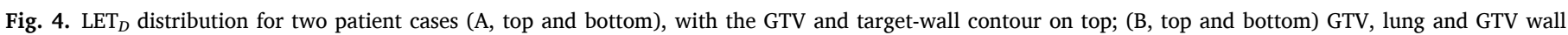
expansion LVHs.

larger GTVs in the cohort and reduced beam path of traversed heterogeneous lung tissue in entrance) above $98 \% \gamma$-PR.

As far for the SFUD plan, the 3 systems under study were highly comparable, with a similar average deviation to experimental data $(<0.1 \%)$ and nearly identical minimum/maximum discrepancies of $\sim 2.5 \% / \sim 2 \%$. For the IMPT plan, FRoG exhibited predictions closest to measurements, on average, out of the 3 engines, while both the gp-MC and RS-MC TPS underestimated by $\sim 1 \%$. With respect to $|\% \Delta|$, gpMC, FRoG and RS-MC TPS performance were in agreement with values of $(3.2 \pm 2.3) \%$, $(3.3 \pm 2.7) \%$ and $(3.4 \pm 2.4) \%$, respectively. Referencing routine patient-specific QA protocol at our facility, these results are clinically acceptable within the 5\%-tolerance level of the mean $|\% \Delta|$ and standard deviation.

In regard to $\mathrm{LET}_{D}$ prediction, FRoG was within a few tenths of keV/ $\mu \mathrm{m}$, compared to reference gp-MC, for both the average and the near-tomaximum $\operatorname{LET}_{D}$. Moreover, results verified that for the selected beam configuration ( 2 orthogonal ports), the $\mathrm{LET}_{D}$ was, on average, $\sim 3 \mathrm{keV} /$ $\mu \mathrm{m}$ in the target and in the tissue nearby, reducing to $\sim 1 \mathrm{keV} / \mu \mathrm{m}$ in the remaining portions of the lung. Maximum $\mathrm{LET}_{D}$ values within and outside the target volume were $\sim 4 \mathrm{keV} / \mu \mathrm{m}$ and $<8 \mathrm{keV} / \mu \mathrm{m}$, respectively.

In this work, FRoG was rigorously benchmarked against dosimetric measurements and gp-MC prediction, as well as in the context of stateof-the-art MC clinical TPSs via end-to-end tests within a thoracic treatment scenario. Recent studies strongly suggest IMPT in the thoracic regions, by means of MC-based calculations, may be necessary to maintain a consistent and acceptable clinical practice for treating lung lesions. Here, we demonstrate that a well-designed analytical PB proton dose engine can effectively predict dose in lung tumors, comparable with gp-MC algorithms, consistent with MC-based clinical TPSs and in good agreement with experimental measurements. This has been verified despite approximations during calculation, e.g. $D_{w}$, and neglecting PB degradation in lung, with the latter recently deemed clinically tolerable in most circumstances [18]. FRoG is an analytical PB-class dose engine with clinically acceptable performance in dose calculation for lung lesions. FRoG predictions are in line with the conclusions reported by [37], promoting the use of analytical calculation methods for rapid plan adaptation with NSCLC treatments.

Furthermore, FRoG uniquely offers MC-validated predictions of patient-specific $\mathrm{LET}_{D}$ distributions. Recently, FRoG is supporting the clinical activity at select Varian@ $\odot$ PT facilities as an auxiliary dose engine and investigations in which aim to establish novel beams and multiion treatment strategies $[38,39]$. Future efforts involve multiinstitutional collaborations to investigate treatment delivery with lung phantoms using heavier ions.

A root cause for the relatively poor performance of PT dose engines in lung phantom studies is the TPS beam model design and execution. Lung treatments can be considered a pinnacle of complexity in PT and therefore, proper dose calculation using analytical methods in heterogeneous anatomy requires sophisticated $\mathrm{PB}$ deformation procedures, e. g. high order PB subdivision, and in turn, propagation and handling of lateral dose penumbra. More specifically, to model PB distortion in lateral heterogeneities, RS v.8, for example, decomposes each spot by 19 beamlets, while FRoG, on the other hand, reconstructs the PB with $\sim 350$ unique beamlets. The issue at hand is simply a computational feat that can most efficiently be performed via GPU-accelerations and similarly, other recently developed systems further demonstrate the potential of accelerated codes, performing both optimization and calculation 
procedures well under a minute [40].

Results from the (15)'s study draw attention to important issues regarding mainstream commercial systems which, for thoracic sites, insufficiently describe lateral dose evolution and substantially undersample the necessary PB decomposition. Not all approaches to dose calculation (analytical or simulation) will be suitable for treating anatomically complex and sensitive cases. Other non-commercial systems, however, using analytical or MC approaches have demonstrated similar accuracy, as FRoG, with significant reductions in calculation time for various anatomic treatment sites [41,42] and may consider following end-to-end tests for TP lung lesions as performed here.

The employment in the clinical routine of benchmarked fast analytical dose engines, such as FRoG, using also log files reporting the actual delivered spots, could be a valid alternative to QA-program relying solely on dose measurements in a homogeneous water-like phantom [43] or to time-consuming measurements with thermoluminescent dosimeters and radiochromic film in anthropomorphic phantoms.

The findings of this work, along with key results from the literature, suggest that to improve confidence in TP for lung cancer, prompt redesign of current commercial analytical approaches is warranted. The authors suggest adoption of FRoG or similar approaches may be necessary to balance speed and accuracy for clinical viability, most feasible through GPU-accelerated architecture.

\section{Conclusions}

For thoracic treatment sites, we provide benchmarks for novel analytical methods to PT dose calculation alongside MC methods, both research and clinical. We provide evidence that it is feasible to reach MC accuracy with a fast analytical approach. Our recently developed analytical PBA demonstrated excellent performance in rigorous testing using a heterogeneous lung phantom, further exemplifying the value of PB model reformation. To improve TP quality, commercial vendors should consider state-of-the-art GPU-accelerated analytical PB models and/or fast MC methods.

Data sharing: Research data are stored in a public repository (The Cancer Imaging Archive-4D Lung Database) [24] and will be shared upon request to the corresponding author.

Funding: This work was supported by German Research Council [DFG-KFO214], Deutsche Krebshilfe [Max-Eder 108876] and intramural funds from National Center for Tumor diseases [NCT3.0_2015.21/22 NCT-PRO and Biodose programs]. The funders had no role in study design, data collection and analysis, decision to publish or preparation of the manuscript.

\section{Declaration of Competing Interest}

The authors declare that they have no known competing financial interests or personal relationships that could have appeared to influence the work reported in this paper.

\section{References}

[1] Han Y. Current status of proton therapy techniques for lung cancer. Radiat Oncol J 2019;37:232-48. https://doi.org/10.3857/roj.2019.00633.

[2] Bush DA, Cheek G, Zaheer S, Wallen J, Mirshahidi H, Katerelos A, et al. High-dose hypofractionated proton beam radiation therapy is safe and effective for central and peripheral early-stage non-small cell lung cancer: results of a 12-year experience at loma linda university medical center. Int $J$ Radiat Oncol Biol Phys 2013;86:964-8. https://doi.org/10.1016/j.ijrobp.2013.05.002.

[3] Nguyen QN, Ly NB, Komaki R, Levy LB, Gomez DR, Chang JY, et al. Long-term outcomes after proton therapy, with concurrent chemotherapy, for stage II-III inoperable non-small cell lung cancer. Radiother Oncol 2015;115:367-72. https:// doi.org/10.1016/j.radonc.2015.05.014.

[4] James SS, Grassberger C, Lu HM. Considerations when treating lung cancer with passive scatter or active scanning proton therapy. Transl Lung Cancer Res 2018;7: 210-5. 10.21037/tlcr.2018.04.01.
[5] Grassberger C, Dowdell S, Lomax A, Sharp G, Shackleford J, Choi N, et al. Motion interplay as a function of patient parameters and spot size in spot scanning proton therapy for lung cancer. Int J Radiat Oncol Biol Phys 2013;86:380-6. https://doi. org/10.1016/j.ijrobp.2013.01.024.

[6] Grassberger C, Daartz J, Dowdell S, Ruggieri T, Sharp G, Paganetti H. Quantification of proton dose calculation accuracy in the lung. Int J Radiat Oncol Biol Phys 2014;89:424-30. https://doi.org/10.1016/j.ijrobp.2014.02.023.

[7] Inoue T, Widder J, van Dijk LV, Takegawa H, Koizumi M, Takashina M, et al. Limited impact of setup and range uncertainties, breathing motion, and interplay effects in robustly optimized intensity modulated proton therapy for stage III nonsmall cell lung cancer. Int J Radiat Oncol Biol Phys 2016;96:661-9. https://doi. org/10.1016/j.ijrobp. 2016.06.2454.

[8] Teoh S, George B, Fiorini F, Vallis KA, Van den Heuvel F. Assessment of robustness against setup uncertainties using probabilistic scenarios in lung cancer: a comparison of proton with photon therapy. Br J Radiol 2020;93:20190584. https://doi.org/10.1259/bjr.20190584.

[9] Lomax AJ. Intensity modulated proton therapy and its sensitivity to treatment uncertainties 1: the potential effects of calculational uncertainties. Phys Med Biol 2008;53:1027-42. https://doi.org/10.1088/0031-9155/53/4/014.

[10] Chang JY, Zhang X, Knopf A, Li H, Mori S, Dong L, et al. Consensus guidelines for implementing pencil-beam scanning proton therapy for thoracic malignancies on behalf of the PTCOG thoracic and lymphoma subcommittee. Int J Radiat Oncol Biol Phys 2017;99:41-50. https://doi.org/10.1016/j.ijrobp.2017.05.014.

[11] Jia X, Schümann J, Paganetti H, Jiang SB. GPU-based fast Monte Carlo dose calculation for proton therapy. Phys Med Biol 2012;57:7783-97. https://doi.org/ 10.1088/0031-9155/57/23/7783.

[12] Schreuder AN, Bridges DS, Rigsby L, Blakey M, Janson M, Hedrick SG, et al. Validation of the RayStation Monte Carlo dose calculation algorithm using a realistic lung phantom. J Appl Clin Med Phys 2019;20:127-37. https://doi.org/ 10.1002/acm2.12777.

[13] Tommasino F, Fellin F, Lorentini S, Farace P. Impact of dose engine algorithm in pencil beam scanning proton therapy for breast cancer. Phys Medica 2018;50:7-12. https://doi.org/10.1016/j.ejmp.2018.05.018.

[14] Langner UW, Mundis M, Strauss D, Zhu M, Mossahebi S. A comparison of two pencil beam scanning treatment planning systems for proton therapy. J Appl Clin Med Phys 2018;19:156-63. https://doi.org/10.1002/acm2.12235.

[15] Taylor PA, Kry SF, Followill DS. Pencil beam algorithms are unsuitable for proton dose calculations in lung. Int J Radiat Oncol Biol Phys 2017;99:750-6. https://doi. org/10.1016/j.ijrobp.2017.06.003.

[16] Widesott L, Lorentini S, Fracchiolla F, Farace P, Schwarz M. Improvements in pencil beam scanning proton therapy dose calculation accuracy in brain tumor cases with a commercial Monte Carlo algorithm. Phys Med Biol 2018;63. https:// doi.org/10.1088/1361-6560/aac279.

[17] Saini J, Traneus E, Maes D, Regmi R, Bowen SR, Bloch C, et al. Advanced Proton Beam Dosimetry Part I: Review and performance evaluation of dose calculation algorithms. Transl Lung Cancer Res 2018;7:171-9. 10.21037/tlcr.2018.04.05.

[18] Baumann KS, Flatten V, Weber U, Lautenschläger S, Eberle F, Zink K, et al. Effects of the Bragg peak degradation due to lung tissue in proton therapy of lung cancer patients. Radiat Oncol 2019;14:1-15. https://doi.org/10.1186/s13014-019-13750 .

[19] Winterhalter C, Zepter S, Shim S, Meier G, Bolsi A, Fredh A, et al. Evaluation of the ray-casting analytical algorithm for pencil beam scanning proton therapy. Phys Med Biol 2019;64. https://doi.org/10.1088/1361-6560/aafe58.

[20] Shirey RJ, Wu HT. Quantifying the effect of air gap, depth, and range shifter thickness on TPS dosimetric accuracy in superficial PBS proton therapy. J Appl Clin Med Phys 2018;19:164-73. https://doi.org/10.1002/acm2.12241.

[21] Mein S, Kopp B, Tessonnier T, Ackermann B, Ecker S, Bauer J, et al. Dosimetric validation of Monte Carlo and analytical dose engines with raster-scanning $1 \mathrm{H}$, 4He, 12C, and $16 \mathrm{O}$ ion-beams using an anthropomorphic phantom. Phys Medica 2019;64:123-31. https://doi.org/10.1016/j.ejmp.2019.07.001.

[22] Choi K, Mein SB, Kopp B, Magro G, Molinelli S, Ciocca M, et al. FRoG-A new calculation engine for clinical investigations with proton and carbon ion beams at CNAO. Cancers (Basel) 2018;10:395. https://doi.org/10.3390/cancers10110395.

[23] Mein S, Choi K, Kopp B, Tessonnier T, Bauer J, Ferrari A, et al. Fast robust dose calculation on GPU for high-precision $1 \mathrm{H}, 4 \mathrm{He}, 12 \mathrm{C}$ and $16 \mathrm{O}$ ion therapy: the FRoG platform. Sci Rep 2018;8:1-12. https://doi.org/10.1038/s41598-018-33194-4.

[24] Clark K, Vendt B, Smith K, Freymann J, Kirby J, Koppel P, et al. The cancer imaging archive (TCIA): maintaining and operating a public information repository. J Digit Imaging 2013;26:1045-57. https://doi.org/10.1007/s10278-013-9622-7.

[25] Mirandola A, Molinelli S, Vilches Freixas G, Mairani A, Gallio E, Panizza D, et al. Dosimetric commissioning and quality assurance of scanned ion beams at the Italian National Center for Oncological Hadrontherapy. Med Phys 2015;42: 5287-300. https://doi.org/10.1118/1.4928397.

[26] Mastella E, Molinelli S, Pella A, Vai A, Maestri D, Vitolo V, et al. 4D strategies for lung tumors treated with hypofractionated scanning proton beam therapy: dosimetric impact and robustness to interplay effects. Radiother Oncol 2020;146: 213-20. https://doi.org/10.1016/j.radonc.2020.02.025.

[27] Maes D, Saini J, Zeng J, Rengan R, Wong T, Bowen SR. Advanced proton beam dosimetry part II: Monte Carlo vs. pencil beam-based planning for lung cancer. Transl Lung Cancer Res 2018;7:114-21. 10.21037/tlcr.2018.04.04.

[28] Fredriksson A, Forsgren A, Hårdemark B. Minimax optimization for handling range and setup uncertainties in proton therapy. Med Phys 2011;38:1672-84. https:// doi.org/10.1118/1.3556559.

[29] Ferrari A, Sala PR, Fasso A, Ranft J. FLUKA: A Multi-Particle Transport Code. Menlo Park, CA: 2005. 10.2172/877507. 
[30] Linz U, editor. Ion Beam Therapy. vol. 320. Berlin, Heidelberg: Springer Berlin Heidelberg; 2012. 10.1007/978-3-642-21414-1.

[31] Mairani A, Böhlen TT, Schiavi A, Tessonnier T, Molinelli S, Brons S, et al. A Monte Carlo-based treatment planning tool for proton therapy. Phys Med Biol 2013;58: 2471-90. https://doi.org/10.1088/0031-9155/58/8/2471.

[32] Fuse H, Fujisaki T, Ikeda R, Hakani Z. Applicability of lung equivalent phantom using the cork with absorbed water in radiotherapeutic dosimetry. Int J Med Phys Clin Eng Radiat Oncol 2018;07:27-34. https://doi.org/10.4236/ ijmpcero.2018.71003.

[33] Low DA, Harms WB, Mutic S, Purdy JA. A technique for the quantitative evaluation of dose distributions. Med Phys 1998;25:656-61. https://doi.org/10.1118/ 1.598248.

[34] Miften M, Olch A, Mihailidis D, Moran J, Pawlicki T, Molineu A, et al. Tolerance limits and methodologies for IMRT measurement-based verification QA: Recommendations of AAPM Task Group No. 218. Med Phys 2018;45:e53-83. $10.1002 / \mathrm{mp} .12810$.

[35] Bauer J, Sommerer F, Mairani A, Unholtz D, Farook R, Handrack J, et al. Integration and evaluation of automated Monte Carlo simulations in the clinical practice of scanned proton and carbon ion beam therapy. Phys Med Biol 2014;59: 4635-59. https://doi.org/10.1088/0031-9155/59/16/4635.

[36] Paganetti H, Jiang H, Parodi K, Slopsema R, Engelsman M. Clinical implementation of full Monte Carlo dose calculation in proton beam therapy. Phys Med Biol 2008; 53:4825-53. https://doi.org/10.1088/0031-9155/53/17/023.
[37] Nenoff L, Matter M, Jarhall AG, Winterhalter C, Gorgisyan J, Josipovic M, et al. Daily adaptive proton therapy: is it appropriate to use analytical dose calculations for plan adaption? Int J Radiat Oncol Biol Phys 2020;107:747-55. https://doi.org/ 10.1016/j.ijrobp.2020.03.036.

[38] Kopp B, Mein S, Dokic I, Harrabi S, Böhlen TT, Haberer T, et al. Development and validation of single field multi-ion particle therapy treatments. Int J Radiat Oncol Biol Phys 2020;106:194-205. https://doi.org/10.1016/j.ijrobp.2019.10.008.

[39] Mein S, Dokic I, Klein C, Tessonnier T, Böhlen TT, Magro G, et al. Biophysical modeling and experimental validation of relative biological effectiveness (RBE) for 4He ion beam therapy. Radiat Oncol 2019;14:1-16. https://doi.org/10.1186/ s13014-019-1295-z.

[40] Matter M, Nenoff L, Meier G, Weber DC, Lomax AJ, Albertini F. Intensity modulated proton therapy plan generation in under ten seconds. Acta Oncol (Madr) 2019;58:1435-9. https://doi.org/10.1080/0284186X.2019.1630753.

[41] da Silva J, Ansorge R, Jena R. Fast pencil beam dose calculation for proton therapy using a double-Gaussian beam model. Front Oncol 2015;5:281. https://doi.org/ 10.3389/fonc. 2015.00281.

[42] Beltran C, Tseung HWC, Augustine KE, Bues M, Mundy DW, Walsh TJ, et al. Clinical Implementation of a Proton Dose Verification System Utilizing a GPU Accelerated Monte Carlo Engine. Int J Part Ther 2016;3:312-9. 10.14338/ijpt-1600011.1 .

[43] Johnson JE, Beltran C, Wan Chan Tseung H, Mundy DW, Kruse JJ, Whitaker TJ, et al. Highly efficient and sensitive patient-specific quality assurance for spotscanned proton therapy. PLoS One 2019;14. 10.1371/journal.pone.0212412. 